\title{
MODELACIÓN DESCRIPTIVA DEL YACIMIENTO SANTA LUCÍA (Zn-Pb-Ba), PINAR DEL RÍO (CUBA)
}

\author{
Ramón Guillermo Pérez-Vázquez ${ }^{1 *}$; Elmidio Estévez-Cruz²; Maite Susana Romero-Espinosa ${ }^{3}$ \\ DOI: http://dx.doi.org/10.18273/revbol.v39n2-2017006@ @) (1)
}

Forma de citar: Pérez-Vázquez, R.G., Estévez-Cruz, E., y Romero-Espinosa, M.S. 2017. Modelación descriptiva del yacimiento Santa Lucía (Zn-Pb-Ba), Pinar del Río (Cuba). Boletín de Geología, 39(2): 83-93.

\section{RESUMEN}

La realización del modelo descriptivo para el yacimiento Santa Lucía, ha sido el objetivo primordial de esta investigación. Para ello se contó con los informes de búsqueda y exploración realizados en el área. Se propone un modelo descriptivo, que no es más que una selección de las características geológicas y tecnológicas de un yacimiento que, en calidad de atributos esenciales e invariantes lo distinguen de otro yacimiento, siendo lo fundamental de todo la génesis de su mineralización principal y secundaria, y que pueden ser utilizadas para su estudio, exploración y evaluación. Para la confección del modelo se escogió la Guía de los Perfiles de la Columbia Británica de Canadá. También se estudiaron dos de las características más comunes en este tipo de yacimiento, la zonalidad geoquímica y el patrón de distribución espacial muy relacionadas con su génesis. Podemos concluir que el proceso fue complejo, influenciado por el régimen de azufre y oxígeno y la migración del canal de ascenso de la mineralización, varías zonas de apertura de la cuenca, colocadas paralelamente y cuyos contenidos de azufre pudo variar en el tiempo. De igual forma la zonalidad geoquímica tiene un comportamiento anómalo, observándose una zonación horizontal de $\mathrm{Zn}-\mathrm{S}-\mathrm{Pb}-\mathrm{Ba}$, contraria a la propuesta realizada en investigaciones anteriores, que pudo ser provocado por un proceso complejo, donde influyen tanto la presencia de distintos canales de ascenso de las soluciones mineralizadoras, así como varios ciclos de degeneración de las salmueras, incluso la migración del canal original o eventos tectónicos posteriores. Finalmente llegamos a la conclusión que todos los cuerpos minerales y manifestaciones del distrito metalogénico forman parte del mismo proceso genético formador de menas. Este trabajo ofrece información para la confección del Mapa Metalogénico 1: 250000 dirigido por el Servicio Geológico de Cuba (IGP).

Palabras clave: Modelo descriptivo, yacimientos minerales, sedex, Cuba.

\section{DESCRIPTIVE MODELING OF SANTA LUCÍA MINERAL DEPOSIT (Zn-Pb-Ba), PINAR DEL RÍO (CUBA)}

\begin{abstract}
The research aims at studying the mineral deposit modeling and specifically, at generating a descriptive model of the Santa Lucia deposit. Previous reports and papers related to the study area were consulted to develop the model. During this research, a descriptive model was developed, which consisted in a selection of the geological and technological characteristics of the deposit, which differentiates it from other deposits types, and that they can be used for their study, exploration and, evaluation. The format of the British Columbia Profiles of Canada was chosen to make the descriptive model. Two typical features of these deposits (geochemical zonation and the spatial distribution pattern), which are related to its formation, were also studied. We can conclude the formation process of Santa Lucía mineral deposit was complex, influenced by the régime of sulfur and oxygen and migration of mineralization feeder channel, vary areas of opening of the basin, placed parallels and contents of sulfur could vary in the time. The geochemistry zonality has an anomalous behavior, being observed a horizontal zonation of $\mathrm{Zn}-\mathrm{S}-\mathrm{Pb}-\mathrm{Ba}$, contrary to proposal of previous researchs, it could be provoked by a complex process where influence the presence of different mineralization feeder channels, as well as several cycles of degeneration of the brines, even the migration of the original feeder channel or tectonic later events. Finally, we reach to the conclusion that all the mineral deposits and targets of the metallogenic district are part of the same ore genetic process. Later tectonic activity, distributed for the area in several nappes with deposits and targets, what was a single mineral deposit. This research offers information to the making of the Metallogenic Map 1: 250000 conducted by Geological Surveys of Cuba (IGP).
\end{abstract}

Keywords: Descriptive modeling, mineral deposit, sedex, Cuba.

\footnotetext{
${ }^{1}$ Universidad Tecnológica de La Habana “José Antonio Echeverría”. La Habana, Cuba. (*) monico@civil.cujae.edu.cu

${ }^{2}$ Universidad de Pinar del Río, Cuba. estevez@upr.edu.cu

${ }_{3}^{3}$ Empresa Nacional de Investigaciones Aplicadas de Cienfuegos, Cuba. maite@eniacfg.co.cu
} 


\section{INTRODUCCIÓN}

El distrito metalogénico Santa Lucía es un ejemplo de región en la que a los yacimientos que en ella se localizan se les han asignado varias génesis en función de las diferentes etapas de prospección y exploración que se han desarrollado. Cada etapa ha cumplido sus objetivos, esclareciendo las características geológicas y mineras de los mismos (Pérez-Vázquez et al., 2015).

Luego de los resultados obtenidos con el trabajo Análisis de Fry en el estudio de la génesis del distrito metalogénico Santa Lucía-Matahambre (Pérez-Vázquez et al., 2015) para establecer la relación espacial y genética que existe entre los diferentes yacimientos y manifestaciones del distrito metalogénico y revelar su dirección preferencial, nos dimos a la tarea de realizar la modelación descriptiva preliminar de uno de los yacimientos más importantes que lo componen, el Yacimiento Santa Lucía.

Los yacimientos minerales representan una extraordinaria concentración de metal formados por procesos magmáticos, magmáticos - hidrotermal, hidrotermal $\mathrm{y} / \mathrm{o}$ stress tectónicos en ambientes geodinámicos tipificados, que se encuentran distribuidos de forma heterogénea, por ellos requieren la conjunción de condiciones muy específicas para formar determinados depósitos minerales que ocuparán áreas muy definidas, que son objetivos prioritarios para las empresas de exploración y que pueden ser económicamente minados (Jenkin et al., 2015; Richards, 2013; Groves et al., 2005; Groves and Bierlein, 2007).

No es sólo un proceso el que da origen a la formación de un yacimiento con valor económico, son muchos y condiciones multivariadas. Así diferentes tipos genéticos de yacimientos ocupan diferentes períodos de tiempo en la historia de la Tierra y diferentes patrones de formación (Groves et al., 2005).

Con el desarrollo científico técnico la demanda de recursos minerales se incrementa constantemente para que la sociedad moderna pueda funcionar. Incluso minerales que años atrás no se utilizaban, como es el caso de los elementos raros y dispersos, hoy son extremadamente necesarios como por ejemplo en la telefonía móvil y los dispositivos electrónicos en general.

Perfeccionar los modelos de yacimientos de minerales, incluida la modelación 3D y la identificación de nuevas clases de yacimientos debe dirigirnos a reevaluar el potencial mineral de regiones que previamente han sido poco exploradas (Lusty and Gunn, 2015; Yadav and Porwal, 2017).

En los últimos treinta años se han publicado numerosos trabajos relacionados con los modelos de yacimientos minerales, la mayoría de los cuales han sido elaborados por especialista norteamericanos y canadienses (Berger et al., 2014; Emsbo, 2009; Emsbo et al., 2016; Mosier et al., 2009; Singer et al., 2009; Ariosa-Iznaga y DíazMartínez, 2001).

La mayoría de los procesos geológicos son recurrentes en la historia geológica y alrededor del planeta. Por tanto, no es sorprendente que los yacimientos minerales que tengan características geológicas y generen mineralizaciones similares estén presentes en escenarios comparables en distintas partes del mundo aún en rocas de diferentes edades.

Los yacimientos minerales que son similares en ese sentido constituyen un "tipo de yacimiento mineral" que se define como: "...yacimientos minerales que comparten una serie de atributos geológicos y contienen minerales particulares o una combinación de ellos de manera tal que estas dos características lo distinguen de otros tipos de yacimientos minerales" (Eckstrand et al., 1996 en Ariosa-Iznaga, 2002).

Los modelos pueden ser simples o complicados, pero en todos los casos deben ser flexibles puesto que con el tiempo se generan nuevos datos y descubrimientos y el geólogo debe estar preparado para transformarlos en concepto o para hacer un cambio en la idea predeterminada. Aún restan por descubrir depósitos pobremente expuestos en superficie, pero las futuras exploraciones requieren mejorar los modelos, que indirectamente van a predecir la ubicación de estos depósitos (Adams and Putnam, 1992; Lusty and Gunn, 2015).

Las necesidades de reconocer y distinguir cuáles factores son esenciales y cuáles son fortuitos para la presencia del yacimiento debe estimular la realización de estudios comparativos críticos de los grupos de yacimientos minerales utilizando todas las herramientas geológicas, geoquímicas, geofísicas y estadísticas a disposición (Proenza y Melgarejo, 1998; Ariosa-Iznaga, 2002; Appold and Monteiro, 2009).

Santa Lucía es un ejemplo de yacimiento que ha sido encuadrado en varios modelos por diferentes autores a lo largo de los años desde su descubrimiento. Esta 
investigación va encaminada a obtener un modelo a partir del conocimiento legado y la información actualizada que se ha podido recuperar, en conjunto con las nuevas técnicas informáticas con las que contamos. De esta forma se busca establecer una guía que sea lo más informativa posible para la búsqueda de yacimientos de este tipo. Se tratará de profundizar en características como la zonalidad geoquímica que resulta de la combinación entre la capacidad de migración diferenciada de estos elementos durante el proceso de dispersión geoquímica y el efecto desigual causado por las condiciones físico-químicas, mecánicas, bioquímicas, etc., causantes de esta dirección de la migración (Nosach, 2009; Gorbachev, 2006). Durante la investigación se propone un modelo descriptivo, que no es más que una selección de las características geológicas y tecnológicas, que en calidad de atributos esenciales e invariantes lo distinguen de otro yacimiento, siendo lo fundamental de toda la génesis de su mineralización principal y secundaria, y que pueden ser utilizadas para su estudio, exploración y, evaluación. También se estudiaron dos de las características más comunes en este tipo de yacimiento, la zonalidad geoquímica y el patrón de distribución espacial muy relacionadas con su génesis.

\section{MATERIALES Y MÉTODOS}

\section{Características Geográfico Geológicas de la Región}

El yacimiento Santa Lucía se encuentra ubicado en los límites del Terreno Guaniguanico, parte noroeste de la provincia de Pinar del Río, Cuba Occidental, según la división política administrativa pertenece al Municipio Minas de Matahambre y se encuentra a $46 \mathrm{Km}$ de la capital provincial (FIGURA 1).

El área presenta un relieve relativamente suave, con un sistema de crestas bajos con un rumbo que oscila entre $\left(35^{\circ}-45^{\circ}\right)$ y crestas laterales bien marcadas con rumbo noreste-suroeste, las elevaciones más altas son: Loma Rogelia (256 m) y Matahambre (243 m) (Pérez y Melgarejo, 1998).

Esta es una región con alto potencial minero por lo tanto el principal renglón económico, en tiempos de explotación, fue la minería. Actualmente la economía está fundamentada en la industria agropecuaria y forestal. También se desarrolla el turismo de playa en Cayo Jutías uno de los atractivos más fascinantes de la región (Beades-González, 1998).
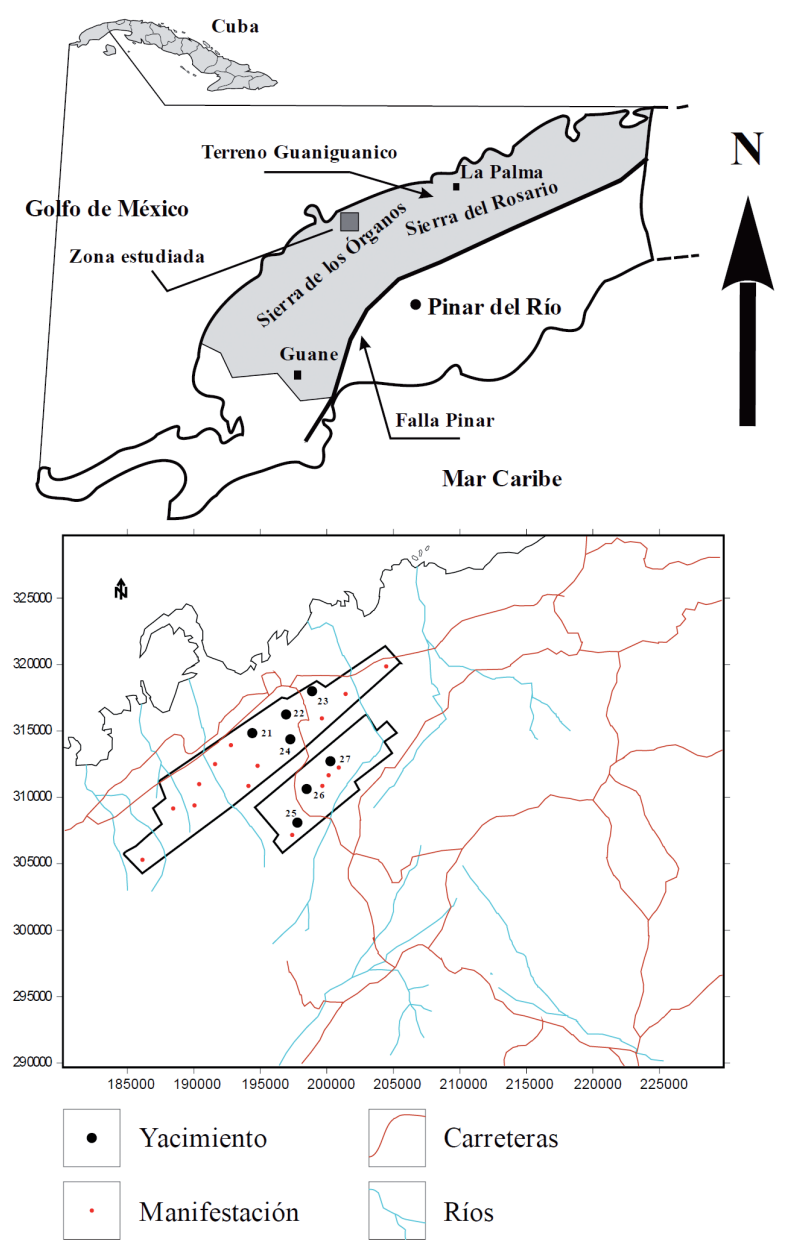

FIGURA 1. Mapa de ubicación geográfica, de yacimientos y manifestaciones en los campos minerales Santa Lucía Castellanos (arriba) y Matahambre (debajo) a escala 1: 50000. 21) La Esperanza; 22) Castellanos; 23) Santa Lucía; 24) Sur; 25) Nieves; 26) Matahambre 27) Mella.

Para la realización del trabajo se contó con los informes de búsqueda y exploración realizados en el área, así como artículos y publicaciones referentes a la misma. En la geología de la región la Formación San Cayetano (Jurásico Inferior-Superior) es la más antigua. Son comunes en ella las estructuras de ripple marks. La base de la Formación es desconocida en ella se encuentran los yacimientos y manifestaciones minerales del área. Se presume que la Formación se formó dentro de un palaeoambiente deltaico, situado en un margen continental pasivo y está compuesta por limolitas y argilitas de color gris a gris oscuro, pardo y violáceas, finamente estratificadas con aspecto pizarroso, intercaladas con areniscas cuarzosas y cuarzo-micáceas, estratificadas de color blanquecino o amarillo rojizo a veces algo oxidado y con espesores variables. También afloran limoareniscas estratificadas de color 
naranja intercaladas con limolitas. En las limolitas aparecen con frecuencia concreciones terrígenas con estructura concéntrica que generalmente oscilan entre 2 y $4 \mathrm{~cm}$ de diámetro. Las limolitas y lutitas pueden contener abundante materia orgánica siendo muy carbonosas y con una coloración gris muy oscuro a negras cuando no están muy meteorizadas. Las rocas que poseen buzamiento monoclinal hacia el NO están complicadas por pequeñas deformaciones locales del plegamiento y por dislocaciones disyuntivas. Juegan un papel determinante en la estructura del yacimiento las dislocaciones disyuntivas longitudinales y transversales (Ovchinnikov et al., 1987).

Conjuntamente con Santa Lucía se encuentran los depósitos Matahambre, Castellanos, La Esperanza, Nieves y más de 20 depósitos y manifestaciones de tipo SEDEX que constituyen el Distrito Metalogénico con contenidos promedios de $1,95 \%$ de $\mathrm{Pb}$ y $6,14 \%$ de $\mathrm{Zn}$ (FIGURA 2).

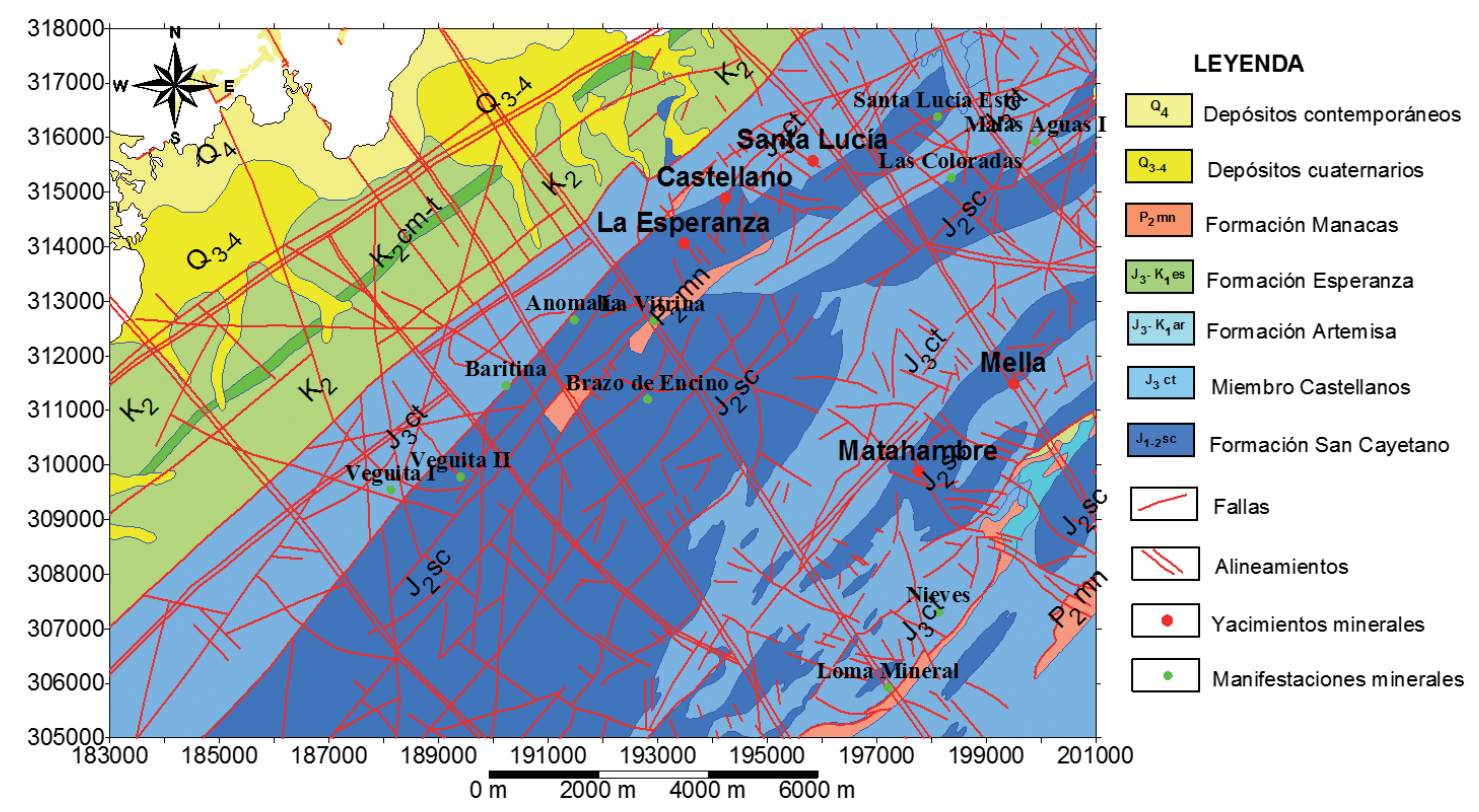

FIGURA 2. Mapa con la geología, de yacimientos y manifestaciones minerales de la región a partir del mapa geológico a escala 1: 50000 (Tomado de Astajov et al., 1985; recopilado y digitalizado por Lastra, 2000).

Los depósitos se caracterizan por ser estratiformes y concordantes con las rocas encajantes. Los cuerpos que afloran a la superficie se encuentran intensamente oxidados y forman amplios sombreros de hierro o gossan, con óxidos e hidróxidos de Fe. De manera general, el sulfuro dominante es la pirita, en menor cuantía la esfalerita y la galena, como minerales acompañantes aparecen la pirrotina, marcasita y calcopirita, como minerales trazas aparecen la arsenopirita, magnetita y la bornita. La zona de oxidación está compuesta por goethita, hematita, magnetita, lepidocrocita, cerusita, anglesita, barita, smithsonita, covelina y calcocita. El cuarzo, la calcita, dolomita, clorita y los feldespatos son los principales minerales no metálicos (Pérez-Vázquez, 2000).

\section{Modelaciones Realizadas en Cuba}

La experiencia acumulada por los geólogos en el transcurso de muchos años de exploración en todo el mundo, ha llevado a los investigadores a la modelación de los yacimientos minerales con el objetivo de determinar las direcciones más racionales de búsqueda. Pero el primer trabajo científicamente argumentado sobre modelos de yacimientos minerales fue elaborado por Erickson (1982). A partir de esta información y la recopilada por Singer (1986), durante la evaluación de los recursos en Alaska se construyeron los antecedentes para la obra fundamental y pionera del Boletín 1693 del USGS "Mineral deposits models" de Cox y Singer en 1986. Mucho más reciente están los trabajos de Emsbo (2009) y Emsbo et al. (2016) sobre los yacimientos SEDEX y el trabajo de Yadav y Porwal (2017) sobre modelo 3D. Antecedieron los modelos de DatosProceso-Criterio (Ludington et al., 1985; Adams and Putnam, 1992), y los del Servicio Geológico de Columbia Británica de Canadá (Lefebure and Ray, 1995; Lefebure and Höy, 1996), son los más utilizados durante la búsqueda y exploración de yacimientos minerales. Ya sea para confeccionar un nuevo modelo o para utilizarlos tal como aparecen, debido a la rica información que ellos brindan. Prosiguen esta labor los Servicios Geológicos 
de Columbia Británica en Canadá, a los que siguieron otros países hasta alcanzar el surgimiento del Programa de Modelación de Yacimientos auspiciado por la Unión Internacional de Ciencias Geológicas -IUGS- y la Comisión de las Naciones Unidas para la Educación, la Ciencia y la Cultura (UNESCO) (Ariosa-Iznaga, 2002).

En nuestro país se han realizado algunas modelaciones entre ellas están las realizada a los metales preciosos y base, del arco Cretácico en el territorio CiegoCamagüey-Las Tunas y para las lateritas niquelíferas en los sectores Pico Galán, Puriales y Minas en la provincia de Guantánamo, al frente de estos trabajos se encontraba el Instituto de Geología y Paleontología. También se realizó la modelación de las lateritas Fe$\mathrm{Ni}-\mathrm{Co}$ en las ofiolitas del Macizo Mayarí-Baracoa Cuba oriental por Ariosa-Iznaga (2002). Ariosa siguió la metodología propuesta por la Guía de Perfiles de la Columbia Británica de Canadá (Lefebure and Ray, 1995; Lefebure and Höy, 1996) y el modelo numérico.

En los últimos años en la región de Sierra de NipeCristal ha constituido una problemática el conocimiento integral de los depósitos de cromo que se encuentran en el territorio que permitan trazar estrategias adecuadas para la realización de los trabajos de prospección geológica. Sobre la base de los resultados de los trabajos de levantamiento, prospección, exploración y los trabajos temáticos realizados en el territorio, fue confeccionado un modelo, el cual parte de la metodología descripta por Ariosa-Iznaga, (2002) sobre modelos descriptivos y la "Guía para Autores de Perfiles de Yacimientos Minerales” de Lefebure y Ray (1995). Díaz et al. (2007) y Njilia et al. (2007) también confeccionaron un modelo descriptivo siguiendo la misma guía de Lefebure y Ray, para los yacimientos de cromita asociados a la faja Mayarí-Baracoa y lo enriquecieron con los modelos de Ley-tonelaje y de probabilidad de ocurrencia, lo cual indica que existe, aunque muy incipiente, el desarrollo de la modelación entre la comunidad geológica de Cuba.

\section{Antecedente de la Génesis y Propuestas de Modelos al Yacimiento Santa Lucía}

En el año 1969 empezó a realizar la exploración preliminar del yacimiento de Santa Lucía y el complejo de trabajos geofísicos-geológicos de búsqueda a escala 1:5000 dentro de los límites del campo menífero de Santa Lucía (Nanián et al., 1978).

Durante los trabajos se describieron las menas del yacimiento como formaciones poligenéticas que se formaron como resultado de tres etapas (Yurpalov et al., 1972). Estas etapas son las siguientes:
Etapa 1 (exhalativo-sedimentaria): Formación del volumen principal de menas piríticas, de plomo-cinc finamente bandeada.

Etapa 2 (hidrotermal?): Formación de menas baríticas de plomo-cinc, menas piríticas, menas baríticas y subetapa independiente de mineralización veti-diseminada polimetálica.

Etapa 3 (hipergénica): Desarrollo de la zona de oxidación y formación del sombrero de hierro.

Siguen desarrollándose investigaciones, con el objetivo encontrar minerales que satisficieran las exigencias de la industria. Se realiza así la búsqueda a escala 1:10 000 entre los años 1979-1982. En esta investigación se concluyó que el yacimiento pertenecía a la clase estratiforme, en esquistos negros, donde la formación de las menas piríticas de plomo cinc tiene un carácter simultáneo con la acumulación de las rocas encajantes en condiciones de cuenca de agua somera, con una considerable contaminación sulfhídrica (Verchigora et al., 1982).

Pero no es hasta que Simón $(1983,1984)$ siguiendo el modelo hipotético para la génesis de un yacimiento de sulfuros masivos de $\mathrm{Pb}-\mathrm{Zn}$ alojados en sedimentos de Large (1980), clasificó el yacimiento Santa Lucía como del tipo SEDEX. La geología de los depósitos SEDEX y stockwork de $\mathrm{Cu}$ en Cuba occidental se ha resumido por Valdés-Nodarse, 1998.

Valdés et al. (1993), aseveran que la mineralización del yacimiento Santa Lucía pertenece al tipo SEDEX y que se encuentra localizada sólo en los estratos de esquistos negros y en menor medida en las arcillas y que contactan con la secuencia silícea y areniscas de la formación San Cayetano. A partir de análisis isotópicos realizados al $\delta^{34} \mathrm{~S}$ de la pirita de yacimiento Santa Lucía es evidente, que el azufre de la pirita proviene de la reducción bacterial, mientras que en el resto de los yacimientos es de origen endógeno. Lo que le da el carácter marino sedimentario a sus menas (TABLA 1).

TABLA 1. Valores de isotopo $\delta^{34} \mathrm{~S}$ para los yacimientos Santa Lucía y Matahambre. (Tomada de Valdés et al., 1993).

\begin{tabular}{lcc}
\hline Muestra No. & Mineral & ${ }^{34} \mathbf{S ~ 0 / 0 0}$ \\
\hline Santa Lucía 1 & Pirita & $-27,5$ \\
Matahambre 1 & Pirita & $-0,5$ \\
Matahambre 2b & Pirita & $+3,0$ \\
Matahambre 2b (dupl.) & Pirita & $+2,2$ \\
Matahambre 3 & Calcopirita & $+1,1$ \\
Matahambre 4 & Calcopirita & +2.8 \\
Matahambre 5b & Calcopirita & $+1,9$ \\
\hline
\end{tabular}




\section{METODOLOGÍA DEL TRABAJO}

La metodología de la investigación que se siguió fue de tipo descriptiva; que permite caracterizar a un objeto o fenómeno mediante la generalización y el análisis de la información geológica, revelando sus rasgos más significativos, regularidades y tendencias. Este tipo de investigación permite establecer relaciones entre el objeto de estudio y otros objetos o fenómenos, comparar, sintetizar características y rasgos comunes entre un conjunto de estos. Para ello se ha recopilado la información disponible; datos, artículos, resultados de trabajos e informes geológicos. A partir del estudio y revisión de la bibliografía se elaboró el marco teórico que sustenta el trabajo. Se establecieron 4 etapas para la realización de la investigación: 1) Revisión bibliográfica en la que se enfatizó en el estudio de los modelos propuestos por Cox y Singer (1986) y los de la BCCGS (1995). 2) Construcción del modelo descriptivo el que se confeccionó a partir de los siguientes pasos: A) Determinación del tipo de yacimiento y sus principales características, B) Comparación con yacimientos análogos, C) Elección de la guía y atributos para confeccionar el modelo. 3) Estudio de la zonalidad geoquímica con los datos del informe de exploración detallada, con información de litología, análisis químico para seis elementos; plomo, cinc, azufre, plata, oro y sulfato de bario, inclinometría y localización de 198 pozos de dicho informe. Durante estos trabajos se determinaron lo siguientes parámetros: Variación de las productividades lineales de los elementos y de sus relaciones, variación de las Productividades volumétricas, variación del coeficiente de contraste de zonalidad de los elementos, variación del índice de zonalidad de los elementos, determinación de la relación $\mathrm{Zn} /(\mathrm{Zn}+\mathrm{Pb})$.

\section{RESULTADOS}

\section{Análisis y Discusión de los Resultados alcanzados}

Según los resultados del análisis de la zonalidad geoquímica y en contradicción con el diagrama propuesto por Large (1980), podemos inferir que la zonalidad geoquímica horizontal que se manifiesta en el yacimiento Santa Lucía es: Zn-S-Pb-Ba desde las zonas más próximas al canal de ascenso, hasta la parte distal del cuerpo. Este comportamiento se manifiesta tanto en los resultados de los coeficientes de contraste de zonalidad como para el índice de zonalidad (FIGURA 3).

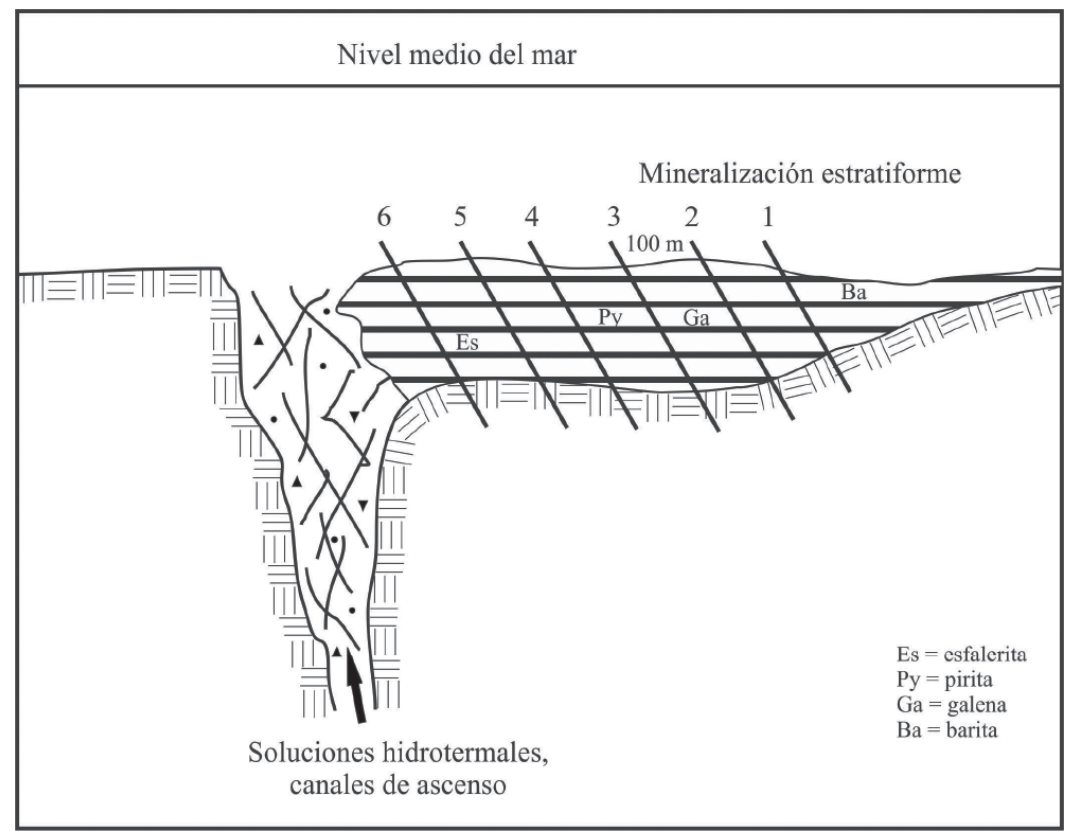

FIGURA 3. Ubicación de los bloques y zonalidad geoquímica horizontal.

El proceso de formación del yacimiento Santa Lucía fue complejo influenciado por el régimen de azufre y oxígeno, incluso la variación de la densidad de la salmuera en el tiempo. Una posible explicación para la variación en la zonalidad geoquímica pudiera ser a partir de la migración del canal de ascenso de la mineralización, varías zonas de apertura de la cuenca, colocadas paralelamente (FIGURA 4). Múltiples períodos de la mineralización durante todo el proceso de formación es una característica de la mayoría de las cuencas sedex, así tenemos por ejemplo los depósitos Mount Isa, Cirque, Hilton o Anarraaq (Emsbo et al., 2016). 


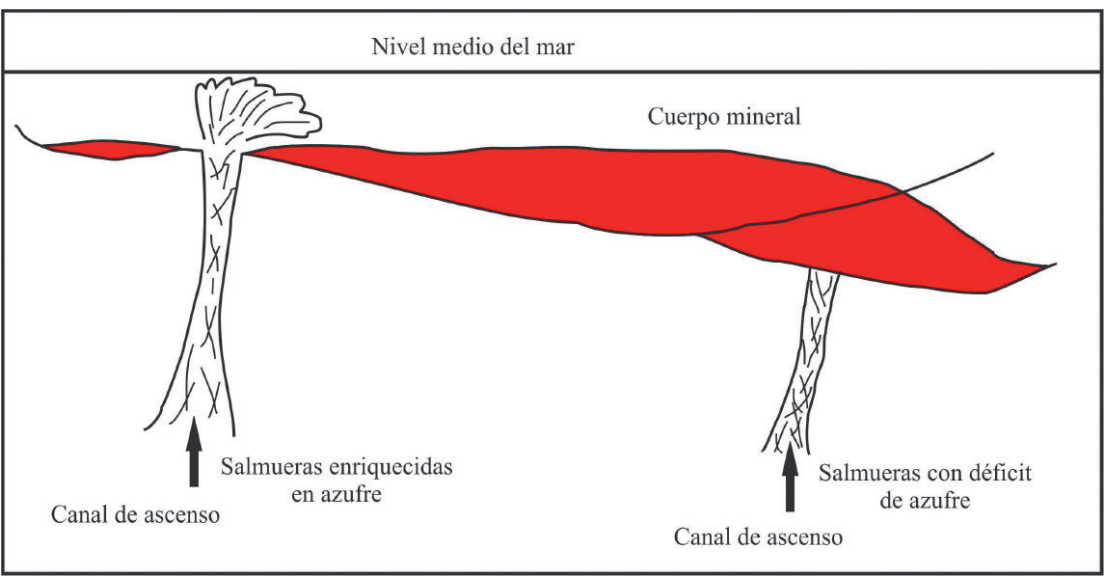

FIGURA 4. Esquema representativo de la presencia de dos canales de ascenso y salmueras con concentraciones diferentes de azufre.

Por otra parte, en el tiempo pudo variar el contenido de azufre de la salmuera que salía por un único canal de ascenso (FIGURA 5). Aunque las características del yacimiento calcopirítico Matahambre con varios filones discordantes con la estratificación y que a medida que aumentaba la profundidad de explotación la mineralización se tornaba más pirrotínica, apunta para varios canales de ascenso de la mineralización con variación de la concentración de azufre. Una potencialmente importante observación es que la mayoría de los depósitos sedex contienen múltiples episodios de mineralización. La cuenca Selwyn por ejemplo, una de las mejores estudiadas, contiene cuatro distintos tipos de eventos de mineralización incluido un pequeño depósito de barita también de tipo sedex (Emsbo et al., 2016).

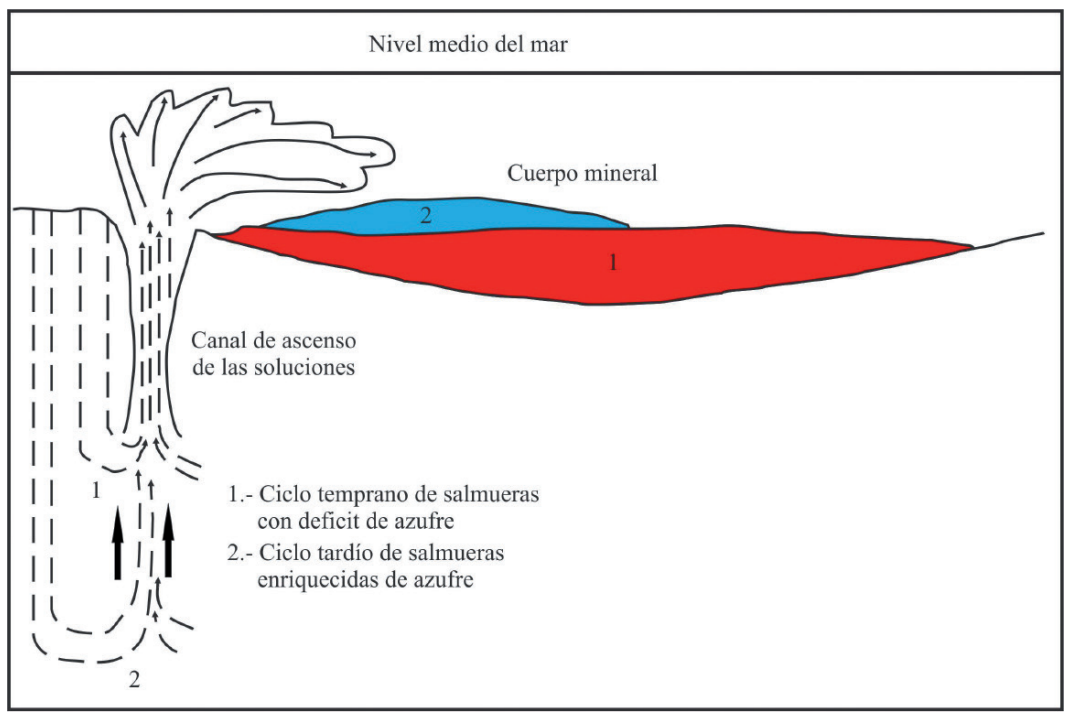

FIGURA 5. Esquema representativo de la presencia de un solo canal por donde circulan salmueras de diferente composición.

En conclusión, la distribución geoquímica del yacimiento es muy irregular, provocado por la propia génesis del mismo y eventos posteriores, observándose una zonalidad geoquímica horizontal de $\mathrm{Zn}-\mathrm{S}-\mathrm{Pb}-\mathrm{Ba}$, contraria a la propuesta por Large (1980).

Los resultados del análisis de Fry para el Campo mineral Santa Lucía revelan que existe una correspondencia entre todos los yacimientos del área; todos se agrupan en una nube de puntos como resultados de un único proceso, que tiene la dirección NE-SW como lo expresa la literatura. De igual forma el análisis de Fry evidencia un bandeamiento o lineamiento, que puede estar producido por fallas, que siguen una dirección $\mathrm{y}$ establece una correspondencia entre todos los yacimientos y manifestaciones, lo que demuestra que están formados por un fenómeno o proceso común (Pérez-Vázquez et al., 2015). 


\section{Modelo Genético}

Finalmente consideramos que todo el proceso de formación se realizó a través de las siguientes etapas (FIGURA 6).

Sedimentos terrígenos deltaicos son depositados en el margen continental pasivo formado con la apertura del mar Caribe (Jurásico Inferior). El agua de mar penetra en el material consolidado (A). Las fallas profundas formadas en el margen continental como

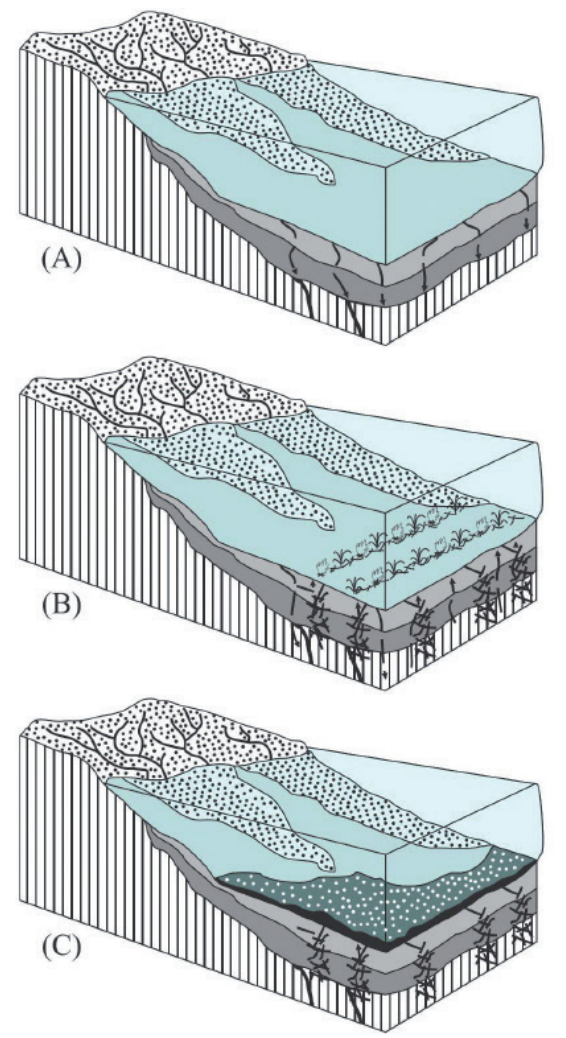

resultado de la apertura, son los canales de filtración y vías de circulación del agua de mar desde las profundidades hasta la superficie, remobilizando los iones contenidos en las rocas e incorporados en todo el proceso (B). Cuando el agua caliente enriquecida en iones metálicos tiene contacto con el azufre presente en el ambiente reductor del fondo de la Cuenca reacciona formando la asociación mineral típica de estos depósitos que con el tiempo se va depositando en el fondo de la cuenca (C).
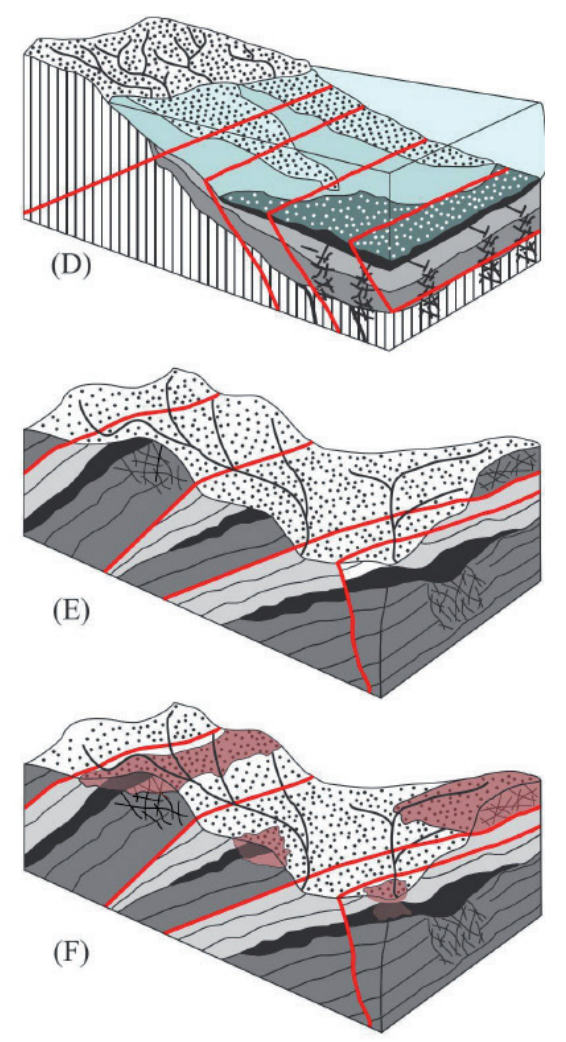

FIGURA 6. Modelo genético de formación del Distrito Metalogénico.

Posteriormente como resultado de los procesos compresivos (Eoceno Inferior-Superior), los cuerpos minerales y las rocas encajantes son expuestas a fallas y movimientos de sobrecorrimiento (D), originando un complejo de mantos de sobrecorrimiento que forman el ambiente tectónico del área dando lugar a los diferentes depósitos que hoy conocemos (E). La continua acción de los agentes de la meteorización, dio paso a la formación de sombreros de hierro. (F). En algunos casos el mismo contiene oro y/o plata con contenidos industriales.

\section{Guías propuestas en el Modelo como resultado de la investigación}

Como ya dijimos para la confección del modelo se tomó la información que aparecía en los informes y artículos revisados para componer un modelo para cada etapa de investigación, utilizando el conocimiento que se tenía en cada momento, posteriormente basados en los datos más recientes y los obtenidos durante el transcurso del tiempo realizar la siguiente propuesta de indicadores del modelo.

\section{A. NOMBRE DEL PERFIL.}

1. Identificación de sinónimos:

2. Productos principales y subproductos: $\mathrm{Pb}, \mathrm{Zn}, \mathrm{Ba}$

3. Ejemplos: Broken Hill, Cannington, Mount Isa, McArthur River (HYC) y Century en Australia, Sullivan en Canadá, Red Dog, Howard Pass en USA y Meggen, Rammelsberg en Alemania. 


\section{B. CARACTERÍSTICAS GEOLÓGICAS.}

1. Descripción resumen:

2. Escenario tectónico:

3. Ambiente de deposición/Escenario geológico:

4. Edad de la mineralización:

5. Tipos de rocas encajantes y asociadas:

6. Forma del yacimiento:

7. Texturas/Estructuras:

8. Mineralogía de la mena (principal y subordinada):

9. Mineralogía de la ganga (principal y subordinada):

10. Mineralogía de las alteraciones:

11. Intemperismo:

12. Controles de las menas:

13. Modelo genético:

14. Tipos de yacimientos asociados:

\section{GUÍAS DE EXPLORACIÓN.}

1. Rasgos geoquímicos:

2. Rasgos geofísicos:

3. Otras guías de exploración

Los yacimientos de este tipo al ser expuestos al intemperismo se oxidan fácilmente dando lugar a la formación de sombreros de hierro o gossan, el cual se enriquece de minerales estables en condiciones exógenas, como lo son la hematita, goethita y limonita, óxidos e hidróxidos de hierro y otros.

\section{FACTORES ECONÓMICOS.}

1. Ley y Tonelaje:

2. Limitantes económicas:

3. Usos finales:

4. Importancia:

\section{CONCLUSIONES}

El proceso de formación del yacimiento Santa Lucía fue complejo influenciado por el régimen de azufre y oxígeno y la migración del canal de ascenso de la mineralización, varías zonas de apertura de la cuenca, colocadas paralelamente y cuyos contenidos de azufre pudo variar en el tiempo.

Existen diversos puntos de vista acerca de la génesis del Campo mineral Santa Lucía, pero la teoría más aceptada es la discutida en el trabajo de exploración detallada de los autores Ovchinnikov et al. (1987).

La zonalidad geoquímica tiene un comportamiento anómalo, observándose una zonación horizontal de
$\mathrm{Zn}-\mathrm{S}-\mathrm{Pb}-\mathrm{Ba}$, contraria a la propuesta por Large en 1980 que pudo ser provocado por un proceso complejo donde influyen tanto la presencia de distintos canales de ascenso de las soluciones mineralizadoras, así como varios ciclos de degeneración de las salmueras, incluso la migración del canal original o eventos tectónicos posteriores.

La zonalidad irregular pudo traer efectos desfavorables o de sobreestimación a la hora de la evaluación del yacimiento.

Todos los cuerpos minerales y manifestaciones del distrito metalogénico forman parte del mismo proceso genético formador de menas. La actividad posterior de la tectónica y mantos de cabalgamiento, distribuyeron por el área en varios depósitos y manifestaciones, lo que fue un solo yacimiento mineral.

\section{AGRADECIMIENTOS}

Los autores desean agradecer especialmente a la Empresa Geominera de Pinar del Río, por su ayuda incondicional en la revisión de los materiales de archivos sin los cuales hubiera sido imposible este trabajo. Al departamento de Geología de la Universidad de Pinar del Río, especialmente al Dr. José Francisco Lastra Rivero quien nos facilitó los materiales sobre geoquímica. A todas aquellas personas que de una forma u otra hicieron posible la realización de este trabajo. A todos MUCHAS GRACIAS.

\section{REFERENCIAS}

Adams, S.S., and Putnam III, B.R. 1992. Application of mineral deposit models in exploration: A case study of sediment-hosted gold deposits, Great Basin, Western United States. Geological Society, London, Special Publications, 63: 1-23.

Appold, M.S., and Monteiro, L.V.S. 2009. Numerical modeling of hydrothermal zinc silicate and sulfide mineralization in the Vazante deposit, Brazil. Geofluids, 9(2): 96-115.

Ariosa-Iznaga, J.D., y Díaz-Martínez, R. 2001. Modelos de yacimiento minerales: Tipologías y aplicaciones. Minería y Geología, 18(2): 3-14.

Ariosa-Iznaga, J.D. 2002. Sobre la problemática del desarrollo de los modelos descriptivos de yacimientos minerales en Cuba. Tesis de Doctorado. Instituto Superior Minero Metalúrgico de Moa, Holguín. 112p. 
Astajov, K., Solianik, V., Vasilev, V., Martínez, D., Fernández de Lara, R., Oubiña, J., Demidov, S., y Santamaría, Z. 1980. Informe sobre los trabajos de levantamiento geológico a escala 1: 50000 en parte noroeste de Pinar del Río (hojas 3484-III - 3483-III 3483-IIIa). Oficina Nacional de Recursos Minerales. La Habana. 497p.

BCCGS. 1995. Mineral Deposit Profiles. 1, 2. British Columbia Canada Geological Survey.

Beades-González, R. 1998. Historia local municipio Minas de Matahambre. Tomo I, Archivo Municipal Minas de Matahambre, Pinar del Río. Informe Inédito, 16p.

Berger, V.I., Mosier, D.L., Bliss, J.D., and Moring, B.C. 2014. Sediment-hosted gold deposits of the worldDatabase and grade and tonnage models. (versión 1.1): U.S. Geological Survey Open-File Report 2014-1074, 46p.

Cox, D., and Singer, D.A. 1986. Mineral deposit models. U.S. Geological Survey Bulletin 1693. 376p.

Díaz-Martínez, R., Njila, T., y Proenza-Fernández, J. 2007. Modelación descriptiva y de ley-tonelaje para pronosticar depósitos de cromitas en Cuba. Minería y Geología, 23(1): 1-32.

Emsbo, P. 2009. Geologic criteria for the assessment of sedimentary exhalative (sedex) $\mathrm{Zn}-\mathrm{Pb}-\mathrm{Ag}$ deposits: U.S. Geological Survey Open-File Report 2009-1209, 21p.

Emsbo, P., Seal, R.R., Breit, G.N., Diehl, S.F., and Shah, A.K. 2016. Sedimentary exhalative (sedex) zinc-leadsilver deposit model: U.S. Geological Survey Scientific Investigations Report 2010-5070-N, 57p.

Erickson, R.L. 1982. Characteristics of mineral deposit occurrences. U.S. Geological Survey Open-File Report 82-795, 250p.

Gorbachev, N.S. 2006. Mineralogical and geochemical zoning and genesis of massive sulfide ores at the Oktyabr'sky deposit. Geology of Ore Deposits. 48(6): 473-488

Groves, D.I., and Bierlein, F.P. 2007. Geodynamic settings of mineral deposit systems. Journal of the Geological Society, 164(1): 19-30.

Groves, D.I., Vielreicher, R.M., Goldfarb. R.J., and Condie, K.C. 2005. Controls on the heterogeneous distribution of mineral deposits through time. Geological Society of London, Special Publications, 248: 71-101.
Jenkin, G.R.T., Lusty, P.A.J., McDonald, I., Smith, M.P., Boyce, A.J., and Wilkinson, J.J. 2015. Ore deposits in an evolving Earth: An introduction. Geological Society of London, 393: 1-8.

Large, D.E. 1980. Geological parameters associated with sediment-hosted, submarine exhalative $\mathrm{Pb}-\mathrm{Zn}$ deposits: An empirical model for mineral exploration. Geologisches Jahrbuch, 40: 59-129.

Lefebure, D.V., and Höy, T. 1996. Selected British Columbia Mineral Deposit Profiles Volume 2: Metallic Deposits. Open file 1996-13, Consultado el 12 de enero de 2017. http://www.em.gov.bc.ca/

Lefebure, D.V, and Ray, G.E. 1995. Selected British Columbia Mineral Deposit Profiles, Volume 1: Metallics and Coal. Open file 1995-20, Consultado el 23 de noviembre de 2016. http://www.em.gov.bc.ca/

Ludington, S., Barton, P.B., and Johnson, K.M. 1985. Mineral Deposit Models: Theory and Practice: U.S. Geological Survey Open-File Report 85-0391, 19p.

Lusty, P.A.J., and Gunn, A.G. 2015. Challenges to global mineral resource security and options for future supply. In: Jenkin, G.R.T., Lusty, P.A.J., McDonald, I., Smith, M.P., Boyce, A.J., and Wilkinson, J.J. (eds). Ore Deposits in an Evolving Earth. Geological Society, London, Special Publications, 393: 265-276.

Mosier, D.L., Berger, V.I., and Singer, D.A. 2009. Volcanogenic massive sulfide deposits of the worlddatabase and grade and tonnage models. U.S. Geological Survey Open-File Report 2009-1034. 50p.

Nanián, B., González, R., y Escalona, H. 1978. Proyecto búsqueda de menas polimetálicas campo mineral Santa Lucía-Castellanos, sectores Sur, Este y Noreste de flancos de la manifestación mineral Sur. 1:25 000. Archivo Provincial Pinar del Río. 102p.

Njilia, T., Díaz, R., y Proenza, J.A. 2007. Los modelos descriptivos, de Ley-Tonelaje y de probabilidad de ocurrencia de los depósitos de cromita asociados a la faja ofiolítica Mayarí-Baracoa. VII Congreso de Geología. Geología y Prospección de Minerales Metálicos. Habana. Cuba. 10p.

Nosach, S. 2009. Aplicación de los métodos geoquímicos en el estudio del yacimiento Mazur, Azov, Ucrania. Ingeniería, Investigación y Desarrollo, 9(2): 57-59.

Ovchinnikov, V., Hernández, A., Robaina, M., Valdivia, M., Rodríguez, A., Salinas, A., Fernández, R., y Estrada, 
N. 1987. Informe sobre los resultados de la exploración detallada del yacimiento polimetálico Santa Lucía de la provincia de Pinar del Río con cálculos de reservas. Oficina Nacional de Recursos Minerales. 441p.

Pérez-Vázquez, R.G., Estévez-Cruz, E., y RomeroEspinosa, M.S. 2015. Análisis de Fry en el estudio de la génesis del distrito metalogénico Santa LucíaMatahambre. Minería y Geología, 31(2): 34-47.

Pérez-Vázquez, R.G. 2000. Pronóstico de metales preciosos (Au y Ag) en el campo mineral Matahambre. Tesis de Maestría. Universidad de Pinar del Río, Cuba. 92p.

Pérez-Vázquez, R.G., y Melgarejo, J.C. 1998. El yacimiento Matahambre (Pinar del Río, Cuba): estructura y mineralogía. Acta Geológica Hispánica, 33(1-4): 133-152.

Proenza, J.A., y Melgarejo, J.C. 1998. Una introducción a la metalogenia de Cuba bajo la perspectiva de la tectónica de placas. Acta Geológica Hispánica, 33(1-4): 89-131.

Richards, J.P. 2013. Giant ore deposits formed by optimal alignments and combinations of geological processes. Nature Geoscience, 6: 911-916.

Simón, A. 1983. El extremo occidental del arco septentrional cubano. Boletín Geociencias, 2(1): 3-27.

Simón, A. 1984. El archipiélago cubano: Ejemplo de metalogenia de los arcos insulares. Oficina Nacional de Recursos Minerales. La Habana. 10p.

Singer, D.A., Berger, V.I., and Moring, B.C. 2009. Sediment-hosted zinc-lead deposits of the world; database and grade and tonnage models. U.S. Geological Survey Open-File Report 2009-1252.

Valdés-Nodarse, E.L., Díaz-Carmona, A., Davies, J., Whitehead, R., and Fonseca, L. 1993. Cogenetic SEDEX Zn-Pb and stockwork $\mathrm{Cu}$ ores, Western Cuba. Exploration and Mining Geology, 2(4): 297-305.

Valdés-Nodarse, E.L. 1998. Pb-Zn SEDEX deposits and their copper stockwork roots, western Cuba. Mineralium Deposita, 33: 560-567.

Verchigora, N., Hernández, A., Simón, A., García, L., y Escalona, N. 1982. Informe de búsqueda a escala 1:10 000 realizado en el campo mineral Castellanos Santa Lucía. Informe final. Oficina Nacional de Recursos Minerales. 508p.
Yadav, S., and Porwal, A. 2017. Controls on SEDEX Mineralization in the Aravalli-Delhi Fold Belt: Insights from Integrated 3D Geological and Geophysical Modelling. Geophysical Research Abstracts, 19: EGU2017-1529.

Yurpalov, O., Zameshikov, M., Sedov, V., Avtoneev, S., Shulzhenke, V., y Amisenov, Y. 1972. Informe con el cálculo de reservas de las menas de plomo-cinc del yacimiento Santa Lucía. Oficina Nacional de Recursos Minerales. 48p.

Trabajo recibido: marzo 24 de 2017

Trabajo aceptado: mayo 03 de 2017

Manuscrito publicado en internet: mayo 05 de 2016 